Article

\title{
Enzyme-Catalyzed Glycosylation of Curcumin and Its Analogues by Glycosyltransferases from Bacillus subtilis ATCC 6633
}

\author{
Yatian Cheng ${ }^{1,+}$, Jian Zhang ${ }^{2,+}$, Yan Shao ${ }^{2}$, Yixiang Xu ${ }^{2}$, Haixia Ge ${ }^{3}$, Boyang $\mathrm{Yu}^{1}{ }^{1}$ \\ and Weiwei Wang ${ }^{1, *}$ \\ 1 Jiangsu Key Laboratory of TCM Evaluation and Translational Research, \\ School of Traditional Chinese Pharmacy, China Pharmaceutical University, Nanjing 211198, China \\ 2 State Key Laboratory of Natural Medicines, China Pharmaceutical University, Nanjing 210009, China \\ 3 School of Life Sciences, Huzhou University, Huzhou 313000, China \\ * Correspondence: weiweiwang@cpu.edu.cn; Tel.: +86-25-8618-5157; Fax: +86-25-8618-5158 \\ + Contributed equally to this work.
}

Received: 18 July 2019; Accepted: 26 August 2019; Published: 29 August 2019

\begin{abstract}
Curcumin is a naturally occurring polyphenolic compound that is commonly used in both medicine and food additives, but its low aqueous solubility and poor bioavailability hinder further clinical applications. For assessing the effect of the glycosylation of curcumin on its aqueous solubility, two glycosyltransferase genes (BsGT1 and BsGT2) were cloned from the genome of the strain Bacillus subtilis ATCC 6633 and over-expressed in Escherichia coli. Then, the two glycosyltransferases were purified, and their glycosylation capacity toward curcumin and its two analogues was verified. The results showed that both BsGT1 and BsGT2 could convert curcumin and its two analogues into their glucosidic derivatives. Then, the structures of the derivatives were characterized as curcumin $4^{\prime}-\mathrm{O}-\beta-\mathrm{D}$-glucoside and two new curcumin analogue monoglucosides namely, curcumoid-O- $\alpha$-D-glucoside (2a) and 3-pentadienone-O- $\alpha$-D-glucoside (3a) by nuclear magnetic resonance (NMR) spectroscopy. Subsequently, the dissolvability of curcumin $4^{\prime}$-O- $\beta$-D-glucoside was measured to be $18.78 \mathrm{mg} / \mathrm{L}$, while its aglycone could not be determined. Furthermore, the optimal catalyzing conditions and kinetic parameters of BsGT1 and BsGT2 toward curcumin were determined, which showed that the Kcat value of BsGT1 was about 2.6-fold higher than that of BsGT2, indicating that curcumin is more favored for BsGT2. Our findings effectively apply the enzymatic approach to obtain glucoside derivatives with enhanced solubility.
\end{abstract}

Keywords: curcumin 4'-O- $\beta$-D-glucoside; glycosyltransferases; Bacillus subtilis ATCC 6633

\section{Introduction}

Curcumin (diferuloylmethane) is extracted from the rhizome of traditional Chinese medicine Zedoary turmeric and Curcuma longa, and is a natural polyphenolic compound that is used as a food coloring in pastries, mustards, curries, and dairy food, as well as rice, meat, and fish dishes in the USA and England [1,2]. Besides these usages, curcumin exerts promising pharmacological properties, such as anti-oxidant, anticancer, anti-inflammatory and antifibrinolytic effects [3-5]. Thus, it has long been believed to be a potential protective natural compound against cardiac diseases, and to possess hepatoprotective and nephroprotective activities [6]. Over the past several decades, extensive research has demonstrated its possible action mechanisms, such as inhibiting experimental allergic encephalomyelitis in the treatment of multicentric sclerosis [7], and as a sarcoplasmic/endoplasmic reticulum calcium pump against cystic fibrosis [8]. However, a few drawbacks of curcumin, including poor pharmacokinetic/pharmacodynamic (PK/PD) properties, low aqueous solubility, and poor 
bioavailability have hindered its further clinical applications [9-11]. While many curcumin analogues have been synthesized in an attempt to unearth new substitutes, none have possessed good drug candidate properties [12]. Thus, this is an imperative issue that has attracted scientists' interest in significantly improving its aqueous solubility.

The glycosylation of natural products (NPs) has the potential to enhance the aqueous solubility of hydrophobic compounds notably, and catalysis by GTs (glycosyltransferases) plays a prominent role in drug discovery and development [13-15]. GTs (EC 2.4.x.y) comprise a great family of enzymes that get involved in the biosynthetic pathways of saccharides and glycoconjugates [16]. Among these, uridine diphosphate-glycosyltransferases (UGTs) can transfer a sugar residue to particular acceptor compounds from an activated nucleotide sugar donor, (for example, uridine diphosphate (UDP)-sugars), forming glycosidic bonds. Currently, the enzymatic synthesis of NP glucosides is more ecofriendly and less time-consuming with higher final-product yields in contrast to chemical synthesis approaches. Therefore, the strategy of enzymatic synthesis of NP glucosides by GTs has been to develop a means to obtain glycosylated small molecules. For example, Bs-YjiC (a GT from Bacillus subtilis 168) could transfer a glucosyl moiety to three particular free $\mathrm{OHs}$ of protopanaxatriol, forming its glucoside derivatives [17], and a UGT from Bacillus licheniformis was elucidated for the glycosylation of phloretin to produce five phloretin glucosides [18]. Moreover, it is encouraging that a fungal GT from Mucor hiemalis exerts the substrate promiscuity of the 72 structurally diverse drug-like natural products [19].

Our previous studies have discovered that Bacillus subtilis ATCC 6633 showed excellent glycosylation capacity toward a few natural products [20]. In this research, we found that the strain B. subtilis ATCC 6633 could convert curcumin to its glucoside derivative. To identify the enzymatic system involved in the glycosylation, two GT genes (BsGT1 and BsGT2) were cloned from B. subtilis ATCC 6633 and over-expressed in Escherichia coli (E. coli). Phylogenetic analysis and sequence alignment with several representative GTs were also investigated. Later, we identified two GTs' glycosylation activity toward curcumin and its two analogues. Then, preparative-scale reactions were conducted, and the glycosylated products of curcumin and its two analogues were isolated and their structures were elucidated. After this, the optimal catalyzing conditions and kinetic parameters of BsGT1 and BsGT2 toward curcumin were determined. Then, the aqueous solubility of the curcumin and its glucoside derivative were measured.

\section{Result}

\subsection{Biotransformation of Curcumin with B. Subtilis ATCC 6633}

Our previous research discovered that B. subtilis ATCC 6633 could convert the pentacyclic triterpenes to their glucoside derivatives [20]. To confirm whether this strain could also transform curcumin, it was cultivated in broth with curcumin, and then, the fermentation liquor was analyzed by high-performance liquid chromatography (HPLC).

Figure 1 shows the HPLC analysis of the 16-h fermentation broth of the strain B. subtilis ATCC $6633 \mathrm{fed}$ with curcumin. In Figure 1, curcumin appears at the retention time (RT) of $29.3 \mathrm{~min}$. After $16 \mathrm{~h}$ of fermentation, a new peak with a RT of 19.3 min appeared. To verify whether the metabolite is the glucoside derivative of curcumin, liquid chromatography-mass spectrometry (LC-MS) analysis was implemented. The results showed that the molecular ion peak of the new peak of the fermentation broth in the presence of curcumin added 162 Da compared with curcumin (shown in Supplementary Materials Figure S1). Consequently, it was concluded that curcumin was transformed by B. subtilis ATCC 6633. 


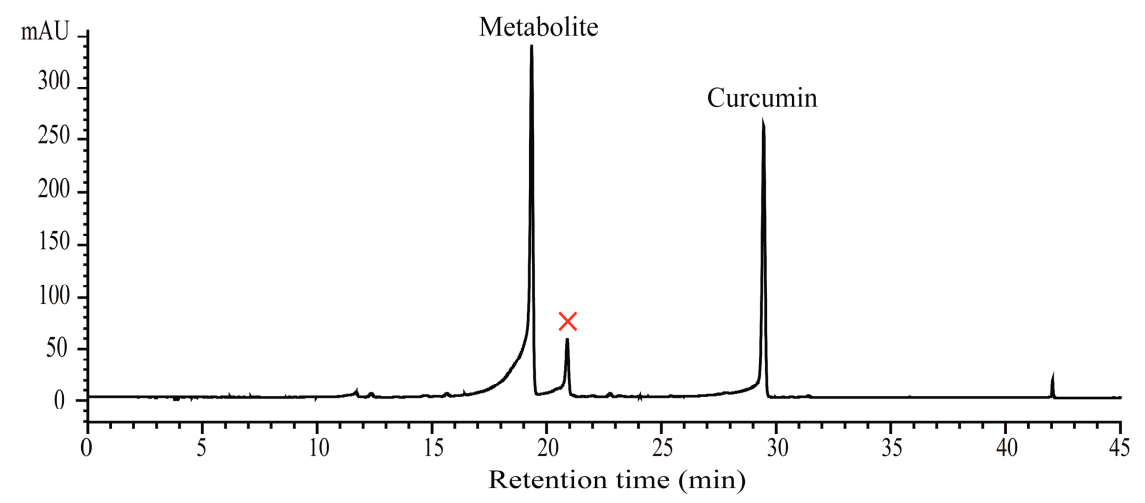

Figure 1. Biotransformation of curcumin by B. subtilis ATCC 6633. The strain was cultivated in potato dextrose culture medium containing curcumin $1.0 \mathrm{mg} / 50 \mathrm{~mL}$. The 16-h cultivations of the fermentation broth were extracted with $50 \mathrm{~mL}$ of ethyl acetate three times, and the organic phase was concentrated and dissolved in methanol for HPLC analysis.

\subsection{Purification of Recombinant BsGT1 and BsGT2}

The recombinant BsGT1 and BsGT2 with His-tags were expressed heterologously in E. coli BL21 and purified through a His-tag Ni-NTA (nitrilotriacetic acid) affinity column. SDS-PAGE analysis indicated that the two purified proteins both showed a molecular mass above the 43-kDa protein marker, which was in good keeping with the sum of the predicted molecular weights of $44.0 \mathrm{kDa}$ for BsGT1 and $44.5 \mathrm{kDa}$ for BsGT2 (Figure 2). The concentration of purified protein was tested by the BCA kit method (Thermo Scientific, China).

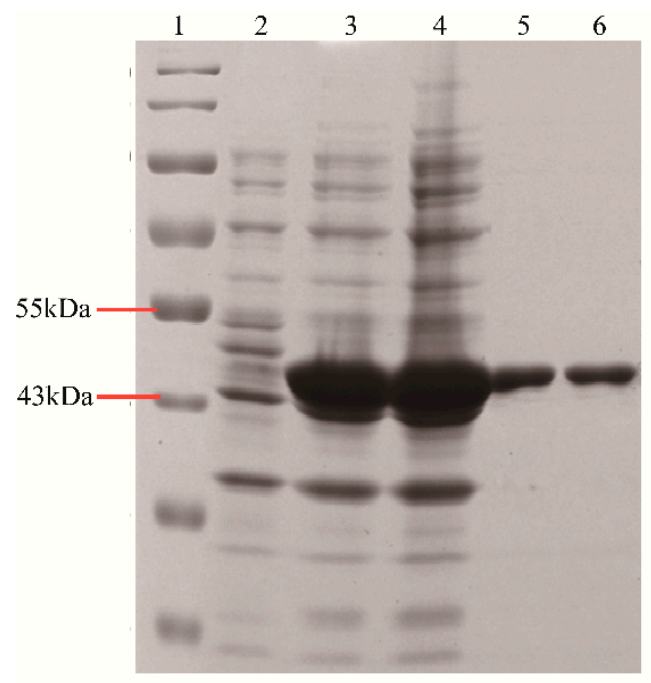

Figure 2. Expression and purification of the recombinant BsGT1 and BsGT2. 1, protein marker; 2, the lysate of E. coli with empty plasmid pET-28a (+); 3, soluble protein of recombinant BsGT1 after induction; 4, soluble protein of recombinant BsGT2 after induction; 5, purified recombinant BsGT1; and, 6, purified recombinant BsGT2.

\subsection{Detection of Glycosylated Products by HPLC-QTOF-MS}

For determining the glycosylation activity of BsGT1 and BsGT2 toward curcumin and its two analogues, the reactions were conducted with $5 \mu \mathrm{g}$ of two purified proteins in $300 \mu \mathrm{L}$ of reaction buffer (50 mM of Tris- $\mathrm{HCl} \mathrm{pH} 8.0$ ) which contained $1.37 \mathrm{mM}$ of UDP-glucose (UDP-Glc) and $2-\mu \mathrm{L}$ substrates $\left(10 \mathrm{mg}\right.$ dissolved in $500 \mu \mathrm{L}$ of DMSO, respectively), and maintained at $37^{\circ} \mathrm{C}$ for $3 \mathrm{~h}$. Then, an extract of each reaction was analyzed by HPLC. All the reactions displayed a new peak at an earlier RT when compared with the substrates (shown in Figures S2 and S3). HPLC-QTOF-MS 
was conducted to further elucidate whether $162 \mathrm{Da}$ was added to different phenolic hydroxyl groups when compared with that of each substrate. As shown in Figure 3, 162 Da was added to Compounds $\mathbf{1 a}\left([\mathrm{M}-\mathrm{H}]^{-}, \mathrm{m} / \mathrm{z}=\sim 529.17\right), \mathbf{2 a}\left([\mathrm{M}-\mathrm{H}]^{-}, \mathrm{m} / \mathrm{z}=\sim 527.23\right)$, and $\mathbf{3 a}\left([\mathrm{M}-\mathrm{H}]^{-}, \mathrm{m} / \mathrm{z}=\sim 487.19\right)$ corresponding to the molecular formulae $\mathrm{C}_{27} \mathrm{H}_{30} \mathrm{O}_{11}, \mathrm{C}_{28} \mathrm{H}_{32} \mathrm{O}_{10}$, and $\mathrm{C}_{25} \mathrm{H}_{28} \mathrm{O}_{10}$, respectively, and these were preliminarily identified as monoglucosides of substrates $\mathbf{1} \sim 3$.

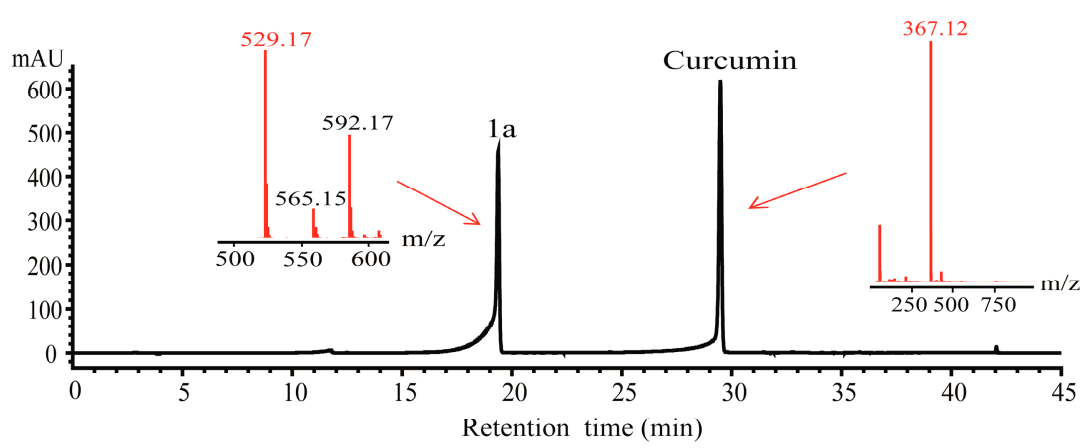

(a)

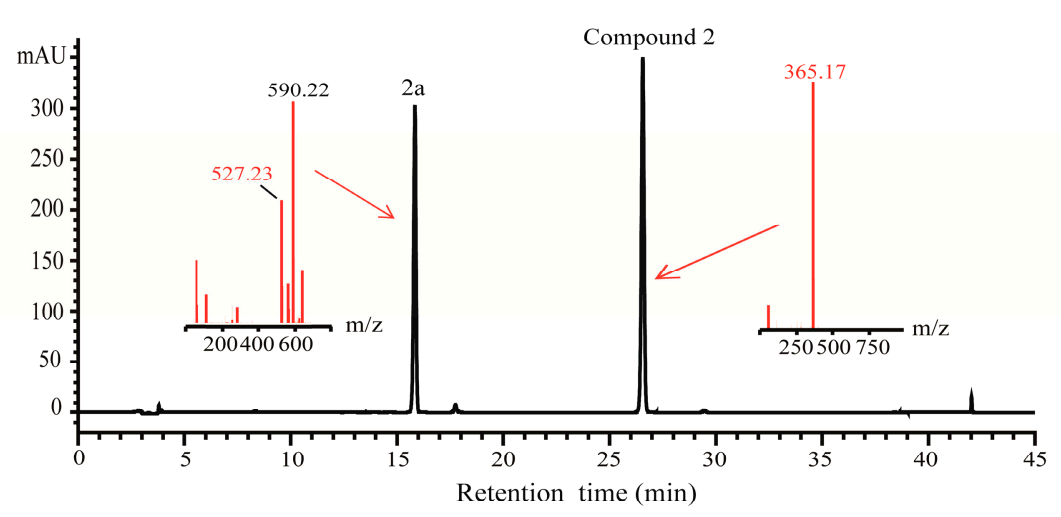

(b)

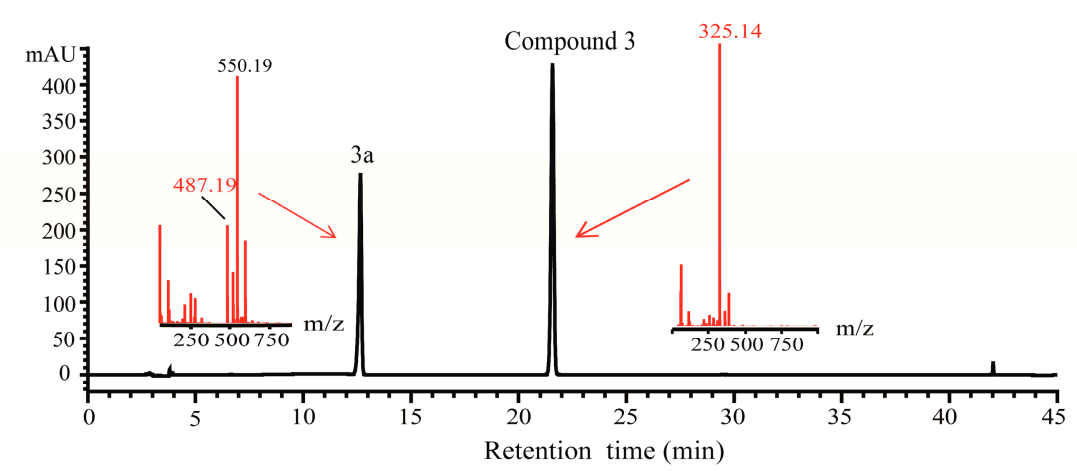

(c)

Figure 3. HPLC analysis of the glucoside derivatives of curcumin $\mathbf{1}(\mathbf{a})$ and its analogues $\mathbf{2}(\mathbf{b})$ and $\mathbf{3}$ (c) catalyzed by BsGT1, and QTOF-MS analysis of those catalyzed by BsGT1 and BsGT2 (red numbers are molecular ion peaks $[\mathrm{M}-\mathrm{H}]^{-}$of glucoside derivatives and substrates).

\subsection{Structural Elucidation of Curcumin and Its Two Analogues Glucoside Derivatives}

For further elucidation of the structures of these Compounds 1a, 2a, and 3a, the purified products were subjected to ${ }^{1} \mathrm{H}$ NMR and ${ }^{13} \mathrm{C}$ NMR analysis, as described in the Supporting Materials (Figures S4-S6). In addition to the signals of the curcumin, and Compounds $\mathbf{1}$ and $\mathbf{2}$ moieties, six carbon signals (from 62.98 to $102.15 \mathrm{ppm}$ of $\mathbf{1 a}$, from 60.78 to $99.95 \mathrm{ppm}$ of $\mathbf{2 a}$, and from 61.22 to $100.12 \mathrm{ppm}$ of $3 \mathrm{a}$ ) belonging to the structure of the glucose moiety were examined. As shown in 
the ${ }^{1} \mathrm{H}$ NMR spectra, the anomeric proton signals at $\delta 5.04(\mathrm{~J}=7.3 \mathrm{~Hz}), 5.26(\mathrm{~J}=4.1 \mathrm{~Hz})$, and 5.26 $(\mathrm{J}=4.8 \mathrm{~Hz})$ of Compounds $\mathbf{1 a}, \mathbf{2} \mathbf{a}$, and $\mathbf{3 a}$, respectively indicated the $\beta$-configuration of Compound $\mathbf{1 a}$ and the $\alpha$-configuration of Compounds $2 \mathbf{a}$ and $3 \mathbf{a}$ for the glucopyranosyl moiety. The ${ }^{13} \mathrm{C}$ NMR spectra showed the glucose anomeric carbon at $\delta 102.15,99.95$, and 100.12 (glycosidation shift), respectively. In addition, the ${ }^{1} \mathrm{H}$ NMR and ${ }^{13} \mathrm{C}$ NMR analysis certified that the structure of Compound 1a was curcumin $4^{\prime}-\mathrm{O}-\beta$-D-glucoside, and the structures of Compounds $2 \mathrm{a}$ and $3 \mathbf{a}$ were O- $\alpha$-D-glucosides. The illustration of the enzyme-catalyzed glycosylation of curcumin, and Compounds 2 and 3 by BsGT1 and BsGT2, are shown in Scheme 1.

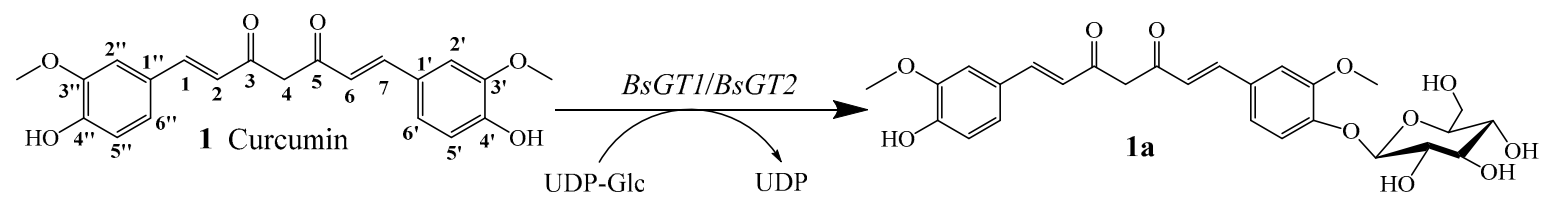

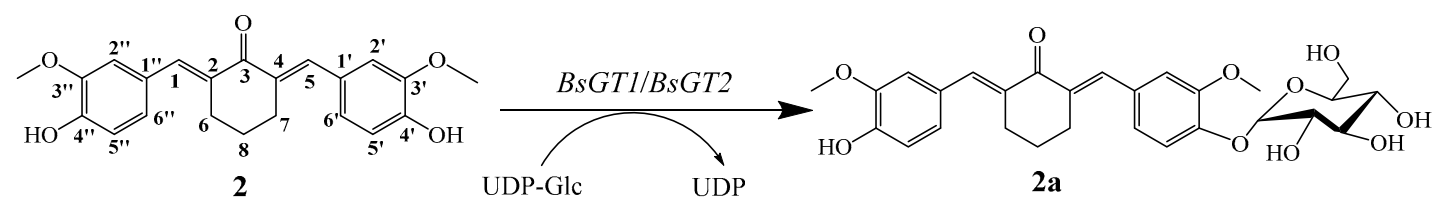

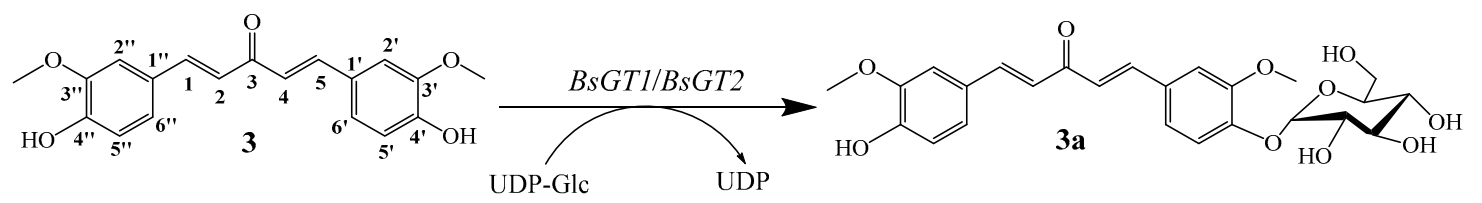

Scheme 1. Catalysis of curcumin and its two analogues by BsGT1/BsGT2. Compound 1a is curcumin $4^{\prime}$-O- $\beta$-D-glucoside.

\subsection{Optimal Catalyzing Conditions and Kinetic Parameters of BsGT1 and BsGT2 toward Curcumin}

In order to determine the optimal catalytic condition by BsGT1 and BsGT2, the influence of temperature, $\mathrm{pH}$ values, and metal ions on enzymatic activity was examined. As shown in Figure 4, both BsGT1 and BsGT2 showed optimal activity at $\mathrm{pH}$ 8.0. The optimum temperatures of BsGT1 and BsGT2 were both $40{ }^{\circ} \mathrm{C}$. However, we found that the two GTs have lower catalytic efficiency at $50{ }^{\circ} \mathrm{C}$. Moreover, they both favored $\mathrm{MnCl}_{2}$ as their cofactor, which was in accordance with GTs using divalent metal ions as cofactors, such as $\mathrm{Mn}^{2+}$ and $\mathrm{Mg}^{2+}$. As shown in the results, the optimal catalyzing conditions for both $B s G T 1$ and $B s G T 2$ are $40{ }^{\circ} \mathrm{C}$ and $\mathrm{pH} 8.0$ with $20 \mathrm{mM}$ of $\mathrm{Mn}^{2+}$.
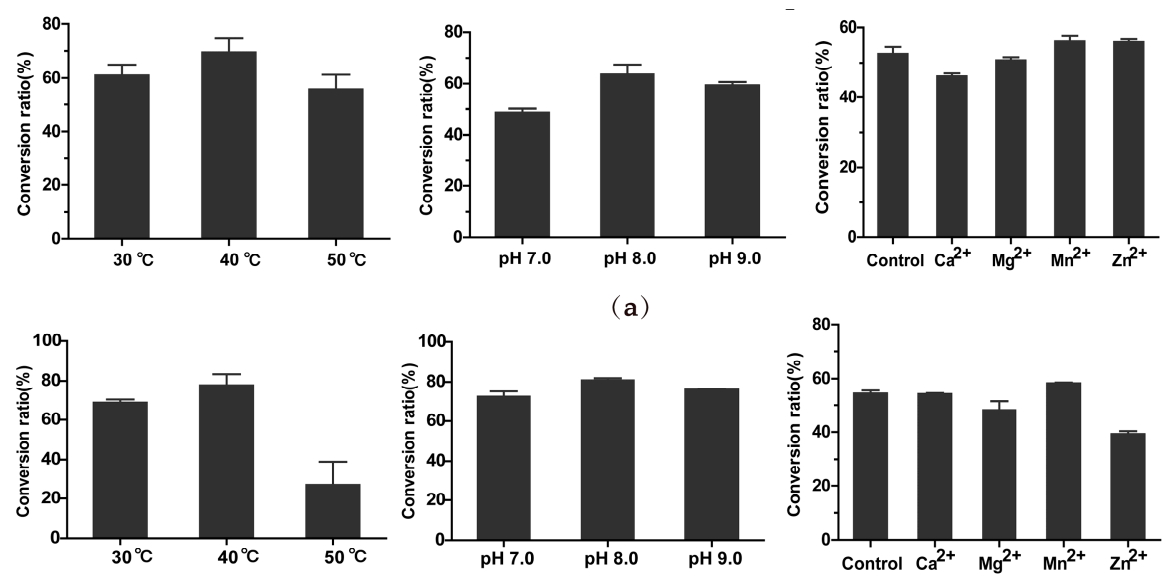

(b)

Figure 4. Effects of temperature, $\mathrm{pH}$, and metal ions on BsGT1 (a) and BsGT2 (b) activity in the biosynthesis of curcumin monoglucoside. The mean $(n=2)$ is shown, and the error bars represent standard deviations. 
The kinetic parameters of purified BsGT1 and BsGT2 toward curcumin were determined as shown in Table 1. The Km values of BsGT1 and BsGT2 for curcumin are $200.1 \pm 23.73 \mu \mathrm{M}$ and $118.3 \pm 17.69 \mu \mathrm{M}$, respectively, indicating that curcumin is more favored for BsGT2 than BsGT1. The Kcat of BsGT1 is about 2.6-fold higher than that of BsGT2.

Table 1. Kinetic parameters of BsGT1 and BsGT2 toward curcumin. GTs: glycosyltransferases.

\begin{tabular}{cccc}
\hline GTs & $\mathbf{K m}(\boldsymbol{\mu M})$ & Kcat $_{\left(\mathbf{S}^{-\mathbf{1}}\right)}$ & $\mathbf{V m a x}\left(\boldsymbol{\mu M} \cdot \mathbf{m i n}^{-\mathbf{1}}\right)$ \\
\hline BsGT1 & $200.1 \pm 23.73$ & $0.47 \pm 0.03$ & $45.31 \pm 2.50$ \\
BsGT2 & $118.3 \pm 17.69$ & $0.18 \pm 0.01$ & $24.87 \pm 1.37$ \\
\hline
\end{tabular}

\subsection{Determination of Solubility of Curcumin and Curcumin $4^{\prime}-O-\beta$-D-glucoside}

The aqueous solubility of curcumin and curcumin $4^{\prime}-\mathrm{O}-\beta$-D-glucoside was examined, and is summarized in Table 2. It is generally accepted that curcumin has extremely poor solubility in water, but that one or more saccharide groups of conjuncted aglycone could improve its aglycone's aqueous solubility. The results revealed that curcumin possessed aqueous solubility after glycosylation.

Table 2. Aqueous solubility of curcumin and curcumin 4'-O- $\beta$-D-glucoside.

\begin{tabular}{cc}
\hline Compound & Aqueous Solubility $(\mathrm{mg} / \mathrm{L})$ \\
\hline Curcumin & N.D \\
Curcumin 4 '-O- $\beta$-D-glucoside & 18.78 \\
\hline \multicolumn{2}{c}{${ }^{1}$ 'N.D' means 'Not detected'. }
\end{tabular}

\subsection{Phylogenetic Analysis and Sequence Alignment}

Phylogenetic analysis of BsGT1, BsGT2, and 20 GTs from plants, bacteria, and fungi was constructed with MEGA 7.0, as shown in Figure 5. The phylogenetic tree divided those GTs into two parts (red numbers 1 and 2), and it is worth considering that the first part includes plant GTs and microbial GTs, which might indicate that BsGT1 and BsGT2 have a closer phylogenetic relationship with some plant UGTs than some microbe ones. To aid further understanding, several representative plant and microbial GTs were aligned based on amino acid sequences.

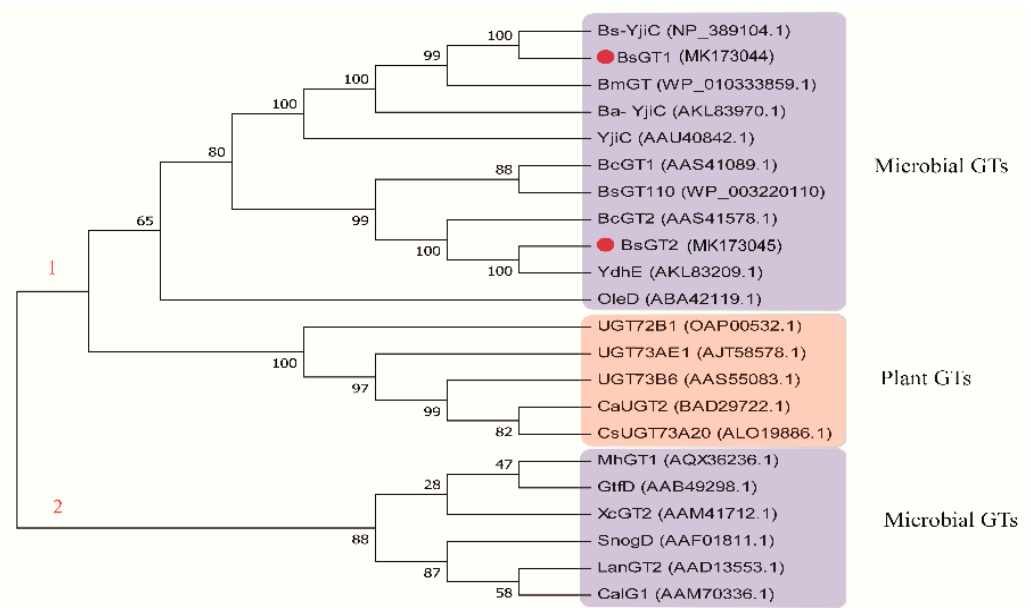

Figure 5. Phylogenetic analysis of $B s G T 1, B s G T 2$, and 20 GTs from plants, bacteria, and fungi. The tree, whose substitution model was Poisson correction, was constructed with the neighbor-joining method based on 1000 bootstrapping cycles. Black numbers at each branch point represent the bootstrap values in the form of percentage. The sequences' GenBank accession numbers are also given. 
Sequence alignment, based on the full-length proteins of BsGT1, BsGT2, and eight selected functionally characterized GTs from plants and bacteria, demonstrated that those microbial GTs showed notably higher homology and similarity with the highly conserved plant secondary product glycosyltransferase (PSPG) motif in plant-derived family 1 GTs [21] (Figure 6). This could give insight into GTs' evolutionary relationship between plants and microbes. Moreover, BsGT1 has a closer phylogenetic relationship with $B s-Y j i C$, which is a promiscuous GT from B. subtilis 168 , showing that it may largely possess substrate flexibility and power regiospecificity.

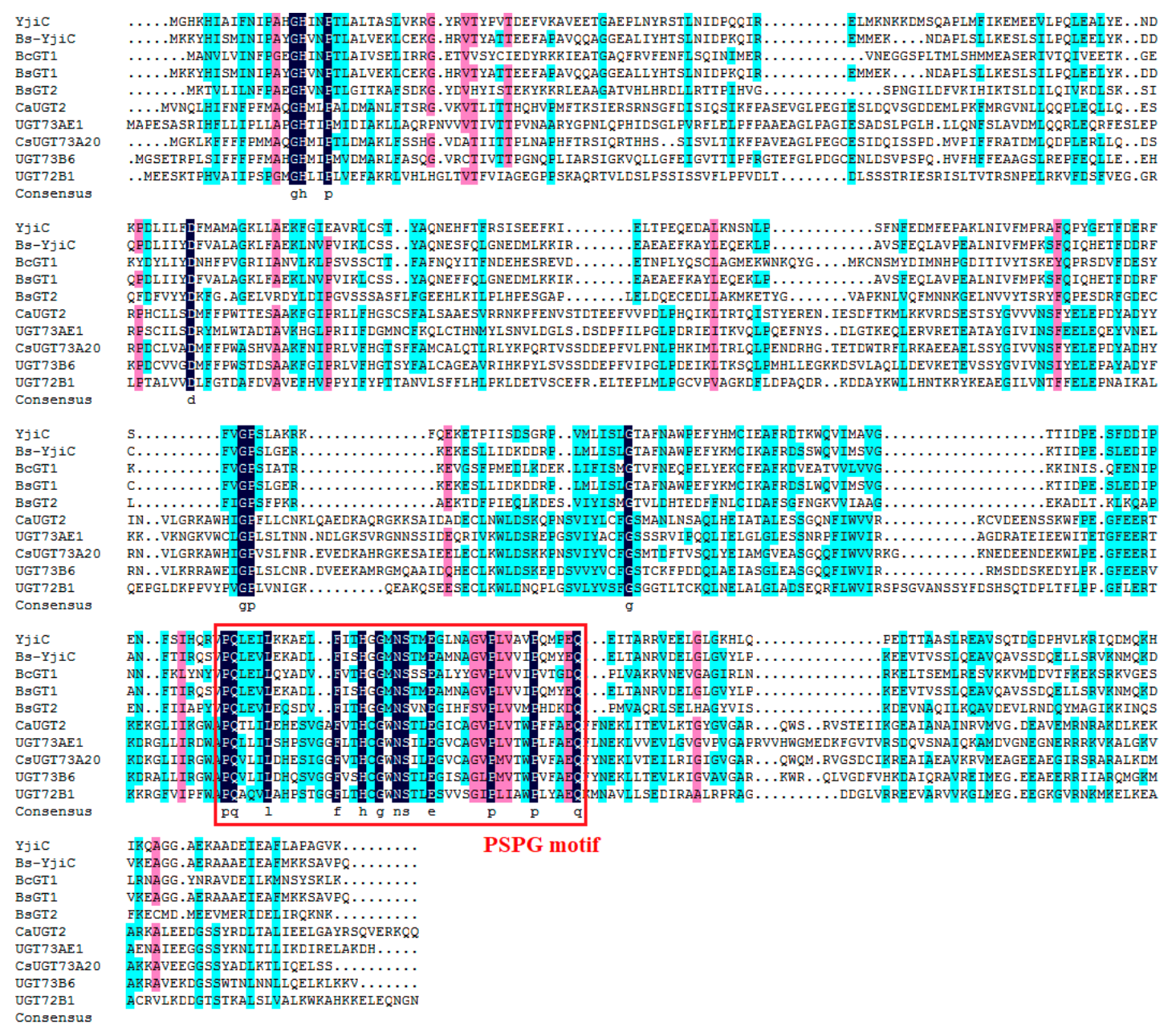

Figure 6. Sequence alignment based on the full-length proteins of BsGT1, BsGT2, and eight GTs from plants and bacteria, and the five GTs from plants are involved in the glycosylation of secondary metabolites. The PSPG box is marked by the red rounded rectangle.

\section{Discussion}

A number of biologically active compounds are glucosides, and even the glycosidic residue plays a central role in their activity; for example, it can improve aglycones' pharmacokinetic parameters and water solubility. Curcumin has been proven to possess multiple bioactivities, but a few drawbacks, including its poor PK/PD properties, low aqueous solubility, or poor bioavailability, hinder further applications. Here, two GTs from B. subtilis ATCC 6633 were cloned and over-expressed to biosynthesize curcumin $4^{\prime}-\mathrm{O}-\beta$-D-glucoside. Then, an aqueous solubility test of curcumin and curcumin $4^{\prime}-\mathrm{O}-\beta-\mathrm{D}$-glucoside indicated that curcumin $4^{\prime}-\mathrm{O}-\beta$-D-glucoside had more preferable aqueous solubility. The pharmacological properties of these biosynthesized glucosides should be further studied, and deep structural analysis of BsGT1 and BsGT2 should also be undertaken to elucidate the difference in catalytic properties. Furthermore, this method could be applied to boost the aqueous solubility 
and bioavailability of other active hydrophobic compounds, and it would be of great interest to introduce the two GTs into glucoside-producing chassis cells to accelerate the production of natural or natural-like active glucosides via metabolic engineering [17].

\section{Materials and Methods}

\subsection{Microorganisms, Plasmids, and Chemicals}

B. subtilis ATCC 6633 was a kind present of Prof. J. P. N. Rosazza of the University of Iowa (Iowa, IA, USA). The two curcumin analogues [22] were synthesized by our laboratory, and could be used as analytical compounds. Curcumin and UDP-glucose (UDP-Glc) were purchased from Aladdin (Aladdin, Shanghai, China). pET-28a (+) was purchased from Novagen (Madison, WI, USA). Other biological reagents were obtained from Takara, except for the His-tag Ni-NTA affinity column, which was purchased from Beyotime (Beyotime, Shanghai, China). HPLC-grade acetonitrile was bought from Tedia Co., Ltd (Fairfield, OH, USA), and E. coli BL21 (DE3) (Transgen Biotech, Beijing, China) was cultivated in Luria Bertani (LB) medium, which contained kanamycin $(100 \mu \mathrm{g} / \mathrm{mL})$. Chemical shifts $(\delta)$ are shown in parts per million (ppm), and coupling constants $(J)$ are quoted in $\mathrm{Hz}$.

\subsection{Identification of Biotransformation Activity of B. subtilis ATCC 6633 to Curcumin}

B. subtilis ATCC 6633 was grown in $50 \mathrm{~mL}$ of potato dextrose culture medium (boiled extraction of $200 \mathrm{~g} / \mathrm{L}$ of peeled potato, $3 \mathrm{~g} / \mathrm{L}$ of $\mathrm{K}_{2} \mathrm{HPO}_{4}, 1.5 \mathrm{~g} / \mathrm{L}$ of $\mathrm{MgSO}_{4}, 10 \mathrm{mg} / \mathrm{L}$ of Vitamin B1, $20 \mathrm{~g} / \mathrm{L}$ of glucose) at $180 \mathrm{rpm}$ and $28^{\circ} \mathrm{C}$. After cultivated for $24 \mathrm{~h}, 1 \mathrm{mg}$ of the curcumin dissolved in $0.1 \mathrm{~mL}$ of dimethyl sulfoxide (DMSO) was added into this medium. Subsequently, the culture was extracted by $50 \mathrm{~mL}$ of ethyl acetate for three times after $16 \mathrm{~h}$ of fermentation. Then, the organic phase was concentrated under vacuum and dissolved in methanol for HPLC analysis in order to assess the biotransformation activity. The identification of biotransformation activity was analyzed by a reverse-phase C18 column $(4.6 \times 250 \mathrm{~mm}, 5 \mu \mathrm{m}$ particles; Welch, Shanghai, China), which was concatenated to an Agilent 1260 HPLC system (ChemStation, B.04.03, Agilent Technologies, Santa Clara, CA, USA) using acetonitrile (solvent $\mathrm{B}$ ) and $0.5 \%$ acetic acid-20 mM ammonium acetate (solvent $\mathrm{D}$ ) as mobile phases. The gradient program was at $20-40 \%$ B for $15 \mathrm{~min}, 40-55 \%$ B for $10 \mathrm{~min}, 55-95 \%$ B for $13 \mathrm{~min}, 95-20 \%$ B for $2 \mathrm{~min}$, and $20 \% \mathrm{~B}$ for $5 \mathrm{~min}$ over $45 \mathrm{~min}$ at a flow rate of $1 \mathrm{~mL} / \mathrm{min}$. The glycosylated products were detected using UV (425 nm) absorbance.

\subsection{Expression and Purification of Recombinant BsGT1 and BsGT2}

The genomic DNA was extracted from B. subtilis ATCC 6633 using a Takara DNAiso Kit (Takara, Dalian, China). Then, the BsGT1 and BsGT2 target genes were amplified from the genomic DNA by PCR, and the primer sets were as follows: forward: $5^{\prime}$-CGGGATCCATGAAAAAGTACCAT ATTTCGA-3', reverse: $5^{\prime}$-CCGCTCGAGTTACTGCGGGACAGCGGATTT-3' ${ }^{\prime}$ for BsGT1, forward: 5' TGAAGACAGTATTGATTTTGA-3' ${ }^{\prime}$, reverse: 5' -CCGCTCGAGTTATTTGTTTTTTTGGCGAAT-3' for BsGT2. Restriction enzyme recognizing sites were designed as BamH I (GGATCC) of the forward primer and Xho I (CTCGAG) of the reverse primer for the two BsGTs. The target genes were subcloned into restriction sites of pET-28a (+) to obtain the expression vector pET-28a-BsGT, whose N-terminal fusion with His-tag allowed the expressed proteins to be purified by a Ni-NTA affinity column. Then, the recombinant vector was introduced into E. coli BL21 (DE3) via heat shock method to form the recombinant $E$. coli.

The recombinant E. coli BL21 strains were cultivated in LB medium $(10 \mathrm{~g} / \mathrm{L}$ of peptone, $5 \mathrm{~g} / \mathrm{L}$ of yeast extract, $10 \mathrm{~g} / \mathrm{L}$ of $\mathrm{NaCl}$ ) at $200 \mathrm{rpm}$ and $37^{\circ} \mathrm{C}$ until the optical density was tested to $0.7-0.8$ at $600 \mathrm{~nm}$. Then, isopropyl- $\beta$-D-thiogalact-opyranoside was added to a final concentration of $0.5 \mathrm{mM}$, and the strains were further cultured at $18^{\circ} \mathrm{C}$ for $16-18 \mathrm{~h}$. The recombinant $E$. coli BL21 strains were harvested through centrifugation ( $8000 \mathrm{rpm}, 10 \mathrm{~min}, 4^{\circ} \mathrm{C}$ ) and resuspended in $20 \mathrm{~mL}$ of lysis buffer (300 $\mathrm{mM}$ of NaCl, $50 \mathrm{mM}$ of $\mathrm{NaH}_{2} \mathrm{PO}_{4}, \mathrm{pH}$ 8.0). Then, the disrupted cells were sonicated on ice. 
After the cell disruption, liquid was centrifuged at $8000 \mathrm{rpm}$ for $10 \mathrm{~min}$ at $4{ }^{\circ} \mathrm{C}$, and the supernatant was loaded onto a pre-equilibrated Ni-NTA column at $4{ }^{\circ} \mathrm{C}$ and washed by washing buffer containing $10-250 \mathrm{mM}$ of imidazole gradient according to the manufacturer's directions. The two target proteins were both eluted by elution buffer, which contained $50 \mathrm{mM}$ of imidazole. Then, the concentrated fractions contained target proteins to $1 \mathrm{~mL}$ using a 15-mL 10-kDa MWCO (Molecular Weight Cut Off) filter (EMD Millipore, San Diego, CA, USA). After desalination, 20\% glycerol was added to the purified proteins solutions, and then they were stored at $-70{ }^{\circ} \mathrm{C}$ or analyzed by SDS-PAGE.

\subsection{Preparative-Scale Reactions and Structural Analysis of the Glucosidic Derivatives}

The preparative-scale reaction was conducted using $500 \mu \mathrm{g}$ of two proteins in $20 \mathrm{~mL}$ of reaction buffer contained $2.46 \mathrm{mM}$ of UDP-Glc, $50 \mathrm{mM}$ of Tris- $\mathrm{HCl} \mathrm{pH} \mathrm{8.0,} 20 \mathrm{mM}$ of $\mathrm{Mn}^{2+}$, and $30 \mathrm{mg}$ of substrate solution (dissolved in DMSO). The reactions were incubated at $30{ }^{\circ} \mathrm{C}$ or $40{ }^{\circ} \mathrm{C}$ for $12 \mathrm{~h}$ and extracted with $20 \mathrm{~mL}$ of ethyl acetate by three times. Then, the organic phase was concentrated and dissolved in $1.5 \mathrm{~mL}$ of methanol, and the substrates and glycosylated products were separated through silica gel column chromatography (200-300 mesh, Marine, Qingdao, China), which were eluted with dichloromethane-methanol (40:1 and 9:1, v/v). The structures of glycosylated products were characterized by ${ }^{1} \mathrm{H}$ NMR and ${ }^{13} \mathrm{C}$ NMR.

The structures were identified using ${ }^{1} \mathrm{H}$ NMR and ${ }^{13} \mathrm{C}$ NMR. The NMR spectra were obtained on either a Bruker AV-500 or Bruker AV-600 spectrometer (Bruker, Billerica, MA, USA) with DMSO- $d 6$ or acetone- $d 6$ as the solvent and TMS (tetramethylsilane) as the internal standard.

\subsection{Enzyme Activity Assay, Optimal Catalyzing Conditions, and Kinetic Parameters of BsGT1 and BsGT2 toward Curcumin}

Enzyme activity assay was conducted with $5 \mu \mathrm{g}$ of two purified proteins in $300 \mu \mathrm{L}$ of reaction buffer ( $50 \mathrm{mM}$ of Tris- $\mathrm{HCl} \mathrm{pH} \mathrm{8.0)} \mathrm{containing} 1.37 \mathrm{mM}$ of UDP-Glc and $2 \mu \mathrm{L}$ of substrates $(10 \mathrm{mg}$ dissolved, respectively, in $500 \mu \mathrm{L}$ of DMSO) and maintained at $37^{\circ} \mathrm{C}$ for $3 \mathrm{~h}$. The assays were quenched by adding $600 \mu \mathrm{L}$ of ethyl acetate for extraction; then, $500 \mu \mathrm{L}$ was drawn and volatilized in a water bath at $55{ }^{\circ} \mathrm{C}$. Subsequently, $200 \mu \mathrm{L}$ of chromatographic pure methanol was added for HPLC and LC-MS analysis, which was performed on Agilent 1260-6530 HPLC-QTOF-MS (Agilent Technologies, Santa Clara, CA, USA). Then, the Agilent 6530 Q-TOF mass spectrometer (Agilent Technologies, Santa Clara, CA, USA) was equipped with an electrospray ionization (ESI) source to implement the MS analysis. Data acquisition and analysis were conducted on an Agilent Mass Hunter Workstation software version B.07.00 (Agilent Technologies, Santa Clara, CA, USA).

To confirm the optimal catalyzing condition of curcumin, the catalytic activity of BsGT1 and BsGT2 at several different temperatures, $\mathrm{pH}$ values, and metal ions were tested. The standard condition was conducted with $5 \mu \mathrm{g}$ of two purified proteins in $300 \mu \mathrm{L}$ of the reaction system, which contained $1.37 \mathrm{mM}$ of UDP-Glc, $50 \mathrm{mM}$ of Tris- $\mathrm{HCl} \mathrm{pH} \mathrm{8.0,20} \mathrm{Mm} \mathrm{of} \mathrm{Mn}^{2+}$, and $2 \mu \mathrm{L}$ of substrate solution (10 mg of substrates dissolved in $500 \mu \mathrm{L}$ of DMSO, respectively) and maintained at $40^{\circ} \mathrm{C}$ for $3 \mathrm{~h}$, and the temperature, $\mathrm{pH}$, or metal ions in the standard condition were replaced by that of the tested condition. For metal ion testing, $20 \mathrm{mM}$ of $\mathrm{MgCl}_{2}, \mathrm{CaCl}_{2}, \mathrm{MnCl}_{2}$, or $\mathrm{ZnCl}_{2}$ was used. For $\mathrm{pH}$ testing, glycine-NaOH buffer ( $\mathrm{pH}$ 9.0) and Tris buffer ( $\mathrm{pH} 7.0$ and 8.0) were used. Then, the reactions were treated by the method described above and analyzed by HPLC. The conversion ratio was calculated by dividing the area of the substrate peaks of the reaction in the HPLC profile by that of the summation of the substrate and product peaks.

The kinetic parameters of BsGT1 and BsGT2 toward curcumin were determined. Kinetic parameters $(300 \mu \mathrm{L})$ toward curcumin in varying concentrations (36 to $360 \mu \mathrm{M})$ were conducted with purified BsGT1 and BsGT2, $50 \mathrm{mM}$ of Tris- $\mathrm{HCl} \mathrm{pH}$ 8.0, $1.37 \mathrm{mM}$ of UDP-Glc, and $2 \mu \mathrm{L}$ of substrates solution. The reactions were incubated for $2 \mathrm{~min}, 4 \mathrm{~min}, 6 \mathrm{~min}, 8 \mathrm{~min}$, and $10 \mathrm{~min}$ at $37^{\circ} \mathrm{C}$, and then terminated by adding $600 \mu \mathrm{L}$ of ethyl acetate. All the subsequent steps were carried out as described above, and the kinetic parameters were calculated by Michaelis-Menten equation analysis utilizing GraphPad 
Prism version 5.01. Besides, the Kcat values were computed utilizing the predicted molecular mass of $44.0 \mathrm{kDa}$ for BsGT1 and $44.5 \mathrm{kDa}$ for BsGT2.

\subsection{Solubility Test}

To determine the solubility of curcumin and its glucoside, each compound was dissolved in $1 \mathrm{~mL}$ of phosphate-buffered saline (PBS) solution at $\mathrm{pH} 7.4$ followed by vortexing for $30 \mathrm{~min}$ and centrifuging at $12,000 \times \mathrm{g}$ for $15 \mathrm{~min}$ at room temperature. Then, aliquots were filtered by a $0.45-\mu \mathrm{m}$ syringe filter, and an equal volume of methanol was added to analyze by HPLC at UV $425 \mathrm{~nm}$. The concentrations of curcumin and its glucoside were measured based on their peak areas utilizing calibration curves determined by the HPLC of authentic samples.

\subsection{Phylogenetic Analysis and Sequence Alignment}

The phylogenetic tree was constructed with MEGA version 7.0 among 20 selected functionally characterized GTs from different species using the neighbor-joining method. After phylogenetic tree analysis, the predicted amino acid sequences of BsGT1 (GenBank accession No. MK173044) and BsGT2 (GenBank accession No. MK173045) were aligned in DNAMAN version 8.0 based on ClustalW multiple alignments. The sequences' GenBank accession numbers and organisms are as shown below: YjiC (AAU40842.1) from Bacillus licheniformis DSM 13, OleD (ABA42119.1) from Streptomyces antibioticus, BcGT1 (AAS41089.1) and BcGT12 (AAS41578.1) from Bacillus cereus ATCC 10987, Bs-YjiC (NP_389104.1) from Bacillus subtilis 168, BsGT110 (WP_003220110) from Bacillus subtilis ATCC 6633, MhGT1 (AQX36236.1) from Mucor hiemalis CGMCC 3.14114, LanGT2 (AAD13553.1) from Streptomyces cyanogenus, GtfD (AAB49298.1) from Amycolatopsis orientalis, SnogD (AAF01811.1) from Streptomyces nogalater, CalG1 (AAM70336.1) from Micromonospora echinospora, CaUGT2 (BAD29722.1) from Catharanthus roseus, UGT73AE1 (AJT58578.1) from Carthamus tinctorius, CsUGT73A20 (ALO19886.1) from Camellia sinensis, UGT73B6 (AAS55083.1) from Rhodiola sachalinensis, UGT72B1 (OAP00532.1) from Arabidopsis thaliana, XcGT2 (AAM41712.1) from Xanthomonas campestris pv. campestris ATCC 33913, BmGT (WP_010333859.1) from Bacillus mojavensis, Ba-YjiC (AKL83970.1) from Bacillus atrophaeus UCMB-5137, BcGT2 (AAS41578.1) from Bacillus cereus ATCC 10987, and YdhE (AKL83209.1) from Bacillus atrophaeus UCMB-5137.

\section{Conclusions}

Studies of microbial GTs have attracted increasing interest and achieved tremendous progress in the enzymatic O-glycosylation of both natural and unnatural compounds, such as ginsenosides [17,20,23,24], antibiotics [25,26], glycopeptides [27,28], flavonoids [18,29-31], and other polyphenols [19,32]. In this study, compared with YjiC from Bacillus licheniformis DSM 13, CaUGT2 from Catharanthus roseus, and UGT76G1 from Stevia rebaudiana catalyzing curcumin to diglucoside and monoglucoside [33-35], BsGT1 and BsGT2 could catalyze curcumin and its two analogues to only their monoglucosides, which indicate that the two GTs are highly selective to the single phenolic hydroxyl group of such compounds. Herein, the two GTs are expected to biosynthesize the monoglucosides of such hydrophobic polyphenol compounds.

Sequence alignment demonstrated that BsGT1 and BsGT2 showed obviously high homology and similarity with the PSPG motif, which can replace plant-derived GTs with biosynthetic natural and natural-like high-value glucosides through overcoming the drawbacks of plant-derived GTs, such as low efficiency in the engineering bacteria. It also provides an effective approach by motif evolution mining the efficient microbial UGTs for application in the biosynthesis of plant-derived rare glucosides. Moreover, microbial GTs are generally more promiscuous than plant-derived GTs toward both the aglycon acceptors and the sugar donors [36], which enables the process of diversification of NPs by conjugating a number of sugar donors to a large range of aglycon acceptors to generate an array of structurally different natural and natural-like products for drug development. In addition, BsGT1 has a closer phylogenetic relationship with Bs-YjiC, which is a promiscuous GT from Bacillus subtilis 
168, indicating that BsGT1 may largely possess similar substrate flexibility and power regiospecificity as that of $B s-Y j i C$, which can be exploited as another effective biocatalyst for the glycosylation of both natural and unnatural products with diverse scaffolds.

Overall, our study provides insights into the enzymatic synthesis of curcumin and its two analogues monoglucosides, expands the enzymatic approach to obtain natural and natural-like glucosides in drug discovery and development, and also provides a useful method to ameliorate the aqueous solubility of active compounds.

Supplementary Materials: The following are available online at http://www.mdpi.com/2073-4344/9/9/734/s1: Figure S1: HPLC-QTOF-MS analysis of curcumin biotransformed by B. subtilis ATCC 6633; Figure S2: Biotransformation of Curcumin 1 (a) and its two analogues 2 (b) and $\mathbf{3}$ (c) by the purified BsGT1; Figure S3: Biotransformation of curcumin 1 (a) and its two analogues 2 (b) and 3 (c) by the purified BsGT2; Figure S4: ${ }^{13} \mathrm{C}$ NMR (a) and ${ }^{1} \mathrm{H}$ NMR (b) analysis of curcumin 4 - $-\mathrm{O}-\beta$-D-glucoside 1a; Figure S5: ${ }^{13} \mathrm{C}$ NMR (a) and ${ }^{1} \mathrm{H}$ NMR (b) analysis of monoglucoside 2a; Figure S6: ${ }^{13} \mathrm{C}$ NMR (a) and ${ }^{1} \mathrm{H}$ NMR (b) analysis of monoglucoside 3a; Figure S7: Kinetic parameters of BsGT1 (a) and BSGT2 (b) toward curcumin.

Author Contributions: Conceptualization, J.Z. and W.W.; methodology, formal analysis and investigation, Y.C., Y.S. and Y.X.; resources, H.G. and B.Y.; writing-original draft preparation, Y.C., J.Z. and W.W.; writing-review and editing, Y.C., J.Z. and W.W.; project administration and supervision, J.Z. and W.W.; funding acquisition, W.W.

Funding: This research was funded by Jiangsu Planned Projects for Postdo ctoral Research Funds awarded to Wei-Wei Wang and the grant number is 2018K085B.

Acknowledgments: For the kind gift of B. subtilis ATCC 6633, we thank Prof. J. P. N. Rosazza of the University of Iowa (USA).

Conflicts of Interest: The authors proclaim no conflict of interest.

\section{References}

1. Scartezzini, P.; Speroni, E. Review on some plants of Indian traditional medicine with antioxidant activity. J. Ethnopharmacol. 2000, 71, 23-43. [CrossRef]

2. Schmandke, H. D-Limonene in citrus fruit with anticarcinogenic action. Ernährungs Umschau 2003, 50, 264-266.

3. Kant, V.; Gopal, A.; Pathak, N.N.; Kumar, P.; Tandan, S.K.; Kumar, D. Antioxidant and anti-inflammatory potential of curcumin accelerated the cutaneous wound healing in streptozotocin-induced diabetic rats. Int. Immunopharmacol. 2014, 20, 322-330. [CrossRef] [PubMed]

4. Vallianou, N.G.; Evangelopoulos, A.; Schizas, N.; Kazazis, C. Potential anticancer properties and mechanisms of action of curcumin. Anticancer Res. 2015, 35, 645-651. [PubMed]

5. Zhang, S.S.; Gong, Z.J.; Li, W.H.; Wang, X.; Ling, T.Y. Antifibrotic effect of curcumin in TGF-beta 1-induced myofibroblasts from human oral mucosa. Asian. Pac. J. Cancer. Prev. 2012, 13, 289-294. [CrossRef] [PubMed]

6. Hashish, E.A.; Elgaml, S.A. Hepatoprotective and Nephroprotective Effect of Curcumin Against Copper Toxicity in Rats. Indian. J. Clin. Biochem. 2016, 31, 270-277. [CrossRef] [PubMed]

7. Aggarwal, B.B.; Harikumar, K.B. Potential therapeutic effects of curcumin, the anti-inflammatory agent, against neurodegenerative, cardiovascular, pulmonary, metabolic, autoimmune and neoplastic diseases. Int. J. Biochem. Cell. Biol. 2009, 41, 40-59. [CrossRef]

8. Lee, W.H.; Loo, C.Y.; Bebawy, M.; Luk, F.; Mason, R.S.; Rohanizadeh, R. Curcumin and its derivatives: Their application in neuropharmacology and neuroscience in the 21st century. Curr. Neuropharmacol. 2013, 11, 338-378. [CrossRef]

9. Burgos-Moron, E.; Calderon-Montano, J.M.; Salvador, J.; Robles, A.; Lopez-Lazaro, M. The dark side of curcumin. Int. J. Cancer. 2010, 126, 1771-1775. [CrossRef]

10. Baell, J.B. Feeling Nature's PAINS: Natural Products, Natural Product Drugs, and Pan Assay Interference Compounds (PAINS). J. Nat. Prod. 2016, 79, 616-628. [CrossRef]

11. Chin, D.; Huebbe, P.; Pallauf, K.; Rimbach, G. Neuroprotective properties of curcumin in Alzheimer's disease-merits and limitations. Curr. Med. Chem. 2013, 20, 3955-3985. [CrossRef] [PubMed]

12. Nelson, K.M.; Dahlin, J.L.; Bisson, J.; Graham, J.; Pauli, G.F.; Walters, M.A. The Essential Medicinal Chemistry of Curcumin. J. Med. Chem. 2017, 60, 1620-1637. [CrossRef] [PubMed]

13. Lee, H.S.; Thorson, J.S. Development of a universal glycosyltransferase assay amenable to high-throughput formats. Anal. Biochem. 2011, 418, 85-88. [CrossRef] [PubMed] 
14. Palcic, M.M. Glycosyltransferases as biocatalysts. Curr. Opin. Chem. Biol. 2011, 15, 226-233. [CrossRef] [PubMed]

15. Thibodeaux, C.J.; Melancon, C.E.; Liu, H.W. Unusual sugar biosynthesis and natural product glycodiversification. Nature 2007, 446, 1008-1016. [CrossRef] [PubMed]

16. Taniguchi, N.; Honke, K.; Fukuda, M.; Narimatsu, H.; Yamaguchi, Y.; Angata, T. Handbook of Glycosyltransferases and Related Genes; Springer: Berlin/Heidelberg, Germany, 2002; pp. 885-903.

17. Dai, L.; Li, J.; Yang, J.; Zhu, Y.; Men, Y.; Zeng, Y.; Cai, Y.; Dong, C.; Dai, Z.; Zhang, X.; et al. Use of a Promiscuous Glycosyltransferase from Bacillus subtilis 168 for the Enzymatic Synthesis of Novel Protopanaxatriol-Type Ginsenosides. J. Agric. Food. Chem. 2018, 66, 943-949. [CrossRef] [PubMed]

18. Pandey, R.P.; Li, T.F.; Kim, E.H.; Yamaguchi, T.; Park, Y.I.; Kim, J.S.; Sohng, J.K. Enzymatic synthesis of novel phloretin glucosides. Appl. Environ. Microbiol. 2013, 79, 3516-3521. [CrossRef]

19. Feng, J.; Zhang, P.; Cui, Y.; Li, K.; Qiao, X.; Zhang, Y.T.; Li, S.M.; Cox, R.J.; Wu, B.; Ye, M.; et al. Regio and Stereo-specific O-Glycosylation of Phenolic Compounds Catalyzed by a Fungal Glycosyltransferase from Mucor hiemalis. Adv. Synth. Catal. 2017, 359, 995-1006. [CrossRef]

20. Wang, W.W.; Xu, S.H.; Zhao, Y.Z.; Zhang, C.; Zhang, Y.Y.; Yu, B.Y.; Zhang, J. Microbial hydroxylation and glycosylation of pentacyclic triterpenes as inhibitors on tissue factor procoagulant activity. Bioorg. Med. Chem. Lett. 2017, 27, 1026-1030. [CrossRef]

21. Lim, E.K. Plant glycosyltransferases: Their potential as novel biocatalysts. Chemistry 2005, 11, 5486-5494. [CrossRef]

22. Ge, H.X.; Chen, L.; Zhang, J.; Kou, J.P.; Yu, B.Y. Inhibitory effect of curcumin analogs on tissue factor procoagulant activity and their preliminary structure-activity relationships. Med. Chem. Res. 2013, 22, 3242-3246. [CrossRef]

23. Luo, S.L.; Dang, L.Z.; Zhang, K.Q.; Liang, L.M.; Li, G.H. Cloning and heterologous expression of UDP-glycosyltransferase genes from Bacillus subtilis and its application in the glycosylation of ginsenoside Rh1. Lett. Appl. Microbiol. 2015, 60, 72-78. [CrossRef] [PubMed]

24. Wang, D.D.; Jin, Y.; Wang, C.; Kim, Y.J.; Perez, Z.E.J.; Baek, N.I.; Mathiyalagan, R.; Markus, J.; Yang, D.C. Rare ginsenoside Ia synthesized from F1 by cloning and overexpression of the UDP-glycosyltransferase gene from Bacillus subtilis: Synthesis, characterization, and in vitro melanogenesis inhibition activity in BL6B16 cells. J. Ginseng. Res. 2018, 42, 42-49. [CrossRef] [PubMed]

25. Wu, C.Z.; Jang, J.H.; Woo, M.; Ahn, J.S.; Kim, J.S.; Hong, Y.S. Enzymatic glycosylation of nonbenzoquinone geldanamycin analogs via Bacillus UDP-glycosyltransferase. Appl. Environ. Microbiol. 2012, 78, 7680-7686. [CrossRef] [PubMed]

26. Liu, Y.; Qin, W.; Liu, Q.; Zhang, J.; Li, H.; Xu, S.; Ren, P.; Tian, L.; Li, W. Genome-wide identification and characterization of macrolide glycosyltransferases from a marine-derived Bacillus strain and their phylogenetic distribution. Environ. Microbiol. 2016, 18, 4770-4781. [CrossRef] [PubMed]

27. Huo, Q.; Li, H.M.; Lee, J.K.; Li, J.; Ma, T.; Zhang, X.; Dai, Y.; Hong, Y.S.; Wu, C.Z. Biosynthesis of Novel Glucosides Geldanamycin Analogs by Enzymatic Synthesis. J. Microbiol. Biotechnol. 2016, 26, 56-60. [CrossRef] [PubMed]

28. Solenberg, P.J.; Matsushima, P.; Stack, D.R.; Wilkie, S.C.; Thompson, R.C.; Baltz, R.H. Production of hybrid glycopeptide antibiotics in vitro and in Streptomyces toyocaensis. Chem. Biol. 1997, 4, 195. [CrossRef]

29. Chiang, C.M.; Wang, T.Y.; Yang, S.Y.; Wu, J.Y.; Chang, T.S. Production of New Isoflavone Glucosides from Glycosylation of 8-Hydroxydaidzein by Glycosyltransferase from Bacillus subtilis ATCC 6633. Catalysts 2018, 8, 387. [CrossRef]

30. Yoon, J.A.; Kim, B.G.; Lee, W.J.; Lim, Y.; Chong, Y.; Ahn, J.H. Production of a novel quercetin glycoside through metabolic engineering of Escherichia coli. Appl. Environ. Microbiol. 2012, 78, 4256-4262. [CrossRef]

31. Gurung, R.B.; Kim, E.H.; Oh, T.J.; Sohng, J.K. Enzymatic synthesis of apigenin glucosides by glucosyltransferase (YjiC) from Bacillus licheniformis DSM 13. Mol. Cells. 2013, 36, 355-361. [CrossRef]

32. Jeon, Y.M. Enzymatic Glycosylation of Phenolic Compounds Using BsGT-3 Based on Molecular Docking Simulation. J. Korean Soc. Appl. Bi. 2009, 52, 98-101. [CrossRef]

33. Gurung, R.B.; Gong, S.Y.; Dhakal, D.; Le, T.T.; Jung, N.R.; Jung, H.J.; Oh, T.J.; Sohng, J.K. Erratum to: Synthesis of Curcumin Glycosides with Enhanced Anticancer Properties Using One-Pot Multienzyme Glycosylation Technique. J. Microbiol. Biotechnol. 2018, 28, 347. [CrossRef] [PubMed] 
34. Kaminaga, Y.; Sahin, F.P.; Mizukami, H. Molecular cloning and characterization of a glucosyltransferase catalyzing glucosylation of curcumin in cultured Catharanthus roseus cells. FEBS Lett. 2004, 567, 197-202. [CrossRef] [PubMed]

35. Dewitte, G.; Walmagh, M.; Diricks, M.; Lepak, A.; Gutmann, A.; Nidetzky, B.; Desmet, T. Screening of recombinant glycosyltransferases reveals the broad acceptor specificity of stevia UGT-76G1. J. Biotechnol. 2016, 233, 49-55. [CrossRef] [PubMed]

36. Song, M.C.; Kim, E.; Ban, Y.H.; Yoo, Y.J.; Kim, E.J.; Park, S.R.; Pandey, R.P.; Sohng, J.K.; Yoon, Y.J. Achievements and impacts of glycosylation reactions involved in natural product biosynthesis in prokaryotes. Appl. Microbiol. Biotechnol. 2013, 97, 5691-5704. [CrossRef] [PubMed]

C 2019 by the authors. Licensee MDPI, Basel, Switzerland. This article is an open access article distributed under the terms and conditions of the Creative Commons Attribution (CC BY) license (http://creativecommons.org/licenses/by/4.0/). 